\title{
Surface Analysis and Electrochemical Studies on Superhydrophobic Aluminium
}

\author{
Subramanian Sathy Srikandan ${ }^{1}$, Swarnambigai B ${ }^{2}$ \\ \{sss.apsc@psgtech.ac.in ${ }^{1}$, bs.apsc@psgtech.ac.in $\left.{ }^{2}\right\}$ \\ Department of applied science, PSG College of Technology, Peelamedu, Coimbatore ${ }^{1}$ \\ Department of Chemistry, PSG College of Technology, Peelamedu, Coimbatore ${ }^{2}$
}

\begin{abstract}
The superhydrophobic surface was prepared on aluminium substrate using wetchemical grafting. The corrosion resistance of the fabricated superhydrophobic surface was compared with bare aluminium surface. The surface morphology of the bare and superhdyrophobic aluminium substrate are examined using scanning electron microscopy method. An attempt is adopted to measure the point of zero charge(pzc) for metallic substrates were determined using contact angle measurement method, since it is economical and easier compared to other methods like X-ray photoelectron Spectrosocopy (XPS). Thermo Gravimetric Analysis (TGA), Zeta potential and Differential Thermal Analysis (DTA) to determine point of zero charge.
\end{abstract}

Keywords: Superhydrophobic aluminium, point of zero charge (pzc), anodization of aluminium, corrosion resistance.

\section{Introduction}

\subsection{Surface hydroxyl groups and point of zero charge}

The Hydroxyl group $\left(\mathrm{OH}^{-}\right)$generally contains oxygen bonded to hydrogen by a covalent bond with a bond length of 0.97 Angstroms.[1,2] These hydroxyl groups are responsible for the wetting property of the substrate.[2] The natural radical $(\mathrm{OH})$ and the negatively charged ion called anion $\left(\mathrm{OH}^{-}\right)$are called hydroxyl ions in common. The empirical formula, electronic state and chemical state of the compound can also be calculated using XPS analysis. The operation cost of this instrument and investment cost are very high. TGA measures the weight loss or the weight gain of the sample with change in temperature and time. There are three types of TGA analysis namely static, dynamic, quasistatic. The temperature stability of the sample can be analysed using this instrument. DTA analysis is used to determine the transition temperature, melting point of the sample. All these characterization are very powerful and they gave very reliable data but the major limitation is they are expensive and can't be used as first hand analysis method. Noh et al, used mass titration method to estimate the point of zero charge of simple oxides. The method found to be simple but the operating procedure found to be complex.[3] Crisiano et al., compared three different methods like: mass titration, poder addition and isoelectric point to determine the pzc of pyrolusite material. Out of the three isoelectric point method found to give results in agreement with literature. They also suggested to use different methods to determine the pzc of a material since all the three 
methods have their own advantages and limitations.[4] Gulicovsk JJ et al., studied the effect of potassium nitrate used during the preparation of alumina suspension. The relationship between particle size of alumina and the $\mathrm{pH}$ were also studied by them.[5] All the above methods are widely adopted by the researchers to estimate the pzc of a material still there is a demand in identifying an easy and user friendly method to calculate the pzc of a solid surface. So, the main objective of the present work is to measure the point of zero charge of the different aluminium substrate using contact angle measurement method. This method can be used to measure the pzc of any solid surface.

\subsection{Surface free energy}

It can be explained as the surplus energy available on the surface, due to the intermolecular force of attraction between the molecules when compared with the remaining bulk of the material.[6-8] The wetting nature decreases with the difference between the polar and dispersive components of liquid and solid molecule values.[8]

Bad wetting

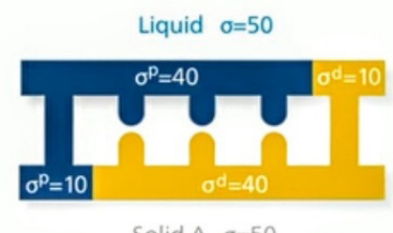

Solid A $\sigma=50$

Bad wetting

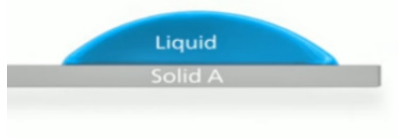

Perfect wetting

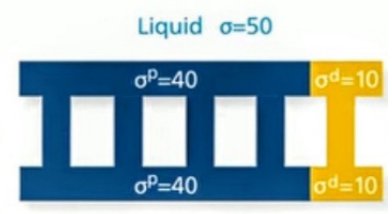

Solid B $\sigma=50$

Perfect wetting

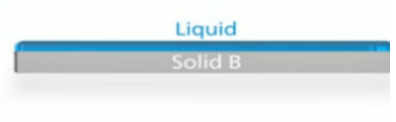

Fig. 1 Schematic representation of bad wetting and perfect wetting.

The following methods are adopted to measure free energy of the surface: i) Fowkees method, ii) New man method, iii) Zisman method and iv) van Osschaudry good method.[6] All these methods give very accurate and precise measurements, but they involve very high initial and operating cost..[9]

\subsection{Wetting}

The surface interaction of the solid and liquid due to intermolecular force of attraction can be defined as wetting.[10] If the adhesive force of solid and liquid is high, the liquid will wet 
the solid surface perfectly.[11] The adhesive and cohesive nature to liquid and solid determines degree of wetting. Based on the static water contact angle (WCA) the surface can

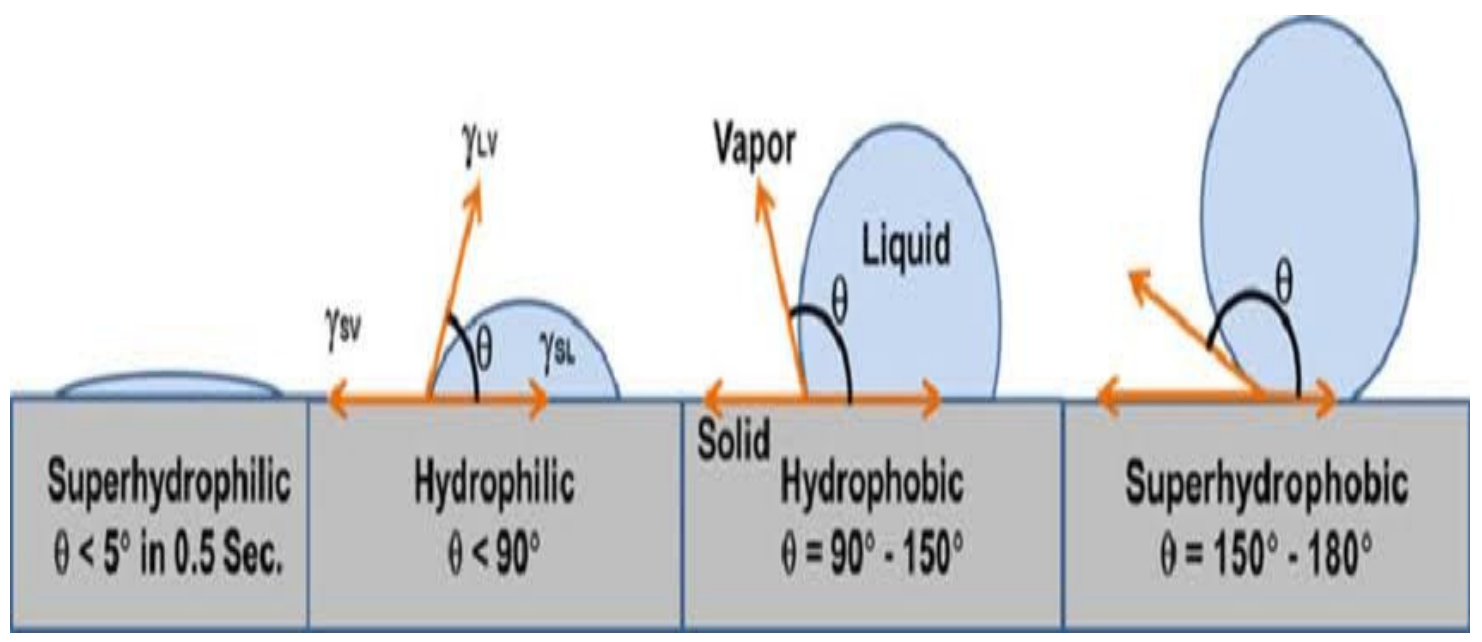

be categorized as shown in Fig. 2.

Fig. 2 Classification of substrate based on static water contact angle (WCA)

\subsection{Point of zero charge (PZC)}

The wetting nature of any liquid is depends on the charge present on the substrate, the charge of the liquid molecule.[12, 13] The location of the ocp (positive or negative) defines the surface charge of the substrate. PZC can be defined as the $\mathrm{pH}$ at which the net charge of the total particle present on the surface is equal to zero, the surface neither attracts nor repels the liquid.[14]

\section{Experimental methods}

\subsection{Anodization of aluminium}

Anodization is an electrochemical process which converts the aluminium substrate into aluminium oxide. The thickness of anodic film formed on the aluminium substrate by altering the current density of the process.[15, 16] In this present work $10 \% \mathrm{w} / \mathrm{v}$ solution of sulphuric acid is used as electrolyte, $\mathrm{Pb}$ sheets are used as cathode and Aluminium sheets (1050 grade) were procured from M/s. Coimbatore metal mart, India having the chemical composition were mainly composed of 1.00 wt.\% of $\mathrm{Si}, 1.95$ wt. $\%$ of $\mathrm{Mg}, 2.50 \mathrm{wt} \%$ of $\mathrm{O}$ and $\mathrm{Al}$ balance is used as anode.

\subsubsection{Hard anodizing}

The bare aluminium substrate was mechanically polished using emery sheets of ascending grade starting from 200, 400 and 600 . The mechanically polished aluminium substrate then 
degreased by immersing in $5 \% \mathrm{NaOH}$ solution for 1 minutes followed by immersing in $5 \%$ solution of dilute nitric acid for 1 minute to remove excess alkali. The chemically cleaned aluminium plates are washed in deionized water and dried in air. The anodization set up was constructed by placing Lead as cathode, aluminium substrate as anode and $10 \% \mathrm{w} / \mathrm{v}$ solution of sulphuric acid as electrolyte. The anodization process was carried out with $2 \mathrm{~A} \mathrm{dm}^{-2}$ current density for 60 minutes. During anodization process aluminium is oxidized as aluminium oxide.[17]

\subsubsection{Sealing of anodized layer}

Hard anodized aluminium substrate is sealed in a boiling water for 20 minutes to convert aluminium oxide into aluminium oxy hydroxide (boehemite - $\mathrm{AlO}(\mathrm{OH})$ ).[18]

\subsubsection{Fabrication of superhydrophobic surface}

The sealed aluminium substrate is immersed in $1 \% \mathrm{w} / \mathrm{v}$ alcoholic solution of lauric acid for 3 hours at $50{ }^{\circ} \mathrm{C}$. The hydroxide groups present on the surface of aluminium substrate are replaced by the laurate group which results SHs.

\subsection{Contact angle measurements}

The liquid-gas interface is contact with the solid surface at some point, using this point the contact angle is measured.[19] The magnitude of wettability of any solid surface can be quantized using contact angle measurements. [20]

\subsection{Electrochemical methods}

Electrochemical impedance spectroscopy (EIS) and Potentiodynamic polarization (Tafel) methods are widely used to determine corrosion efficiency of a material in a highly corrosive environment. The corrosion resistance of a material and its wettability has a close relationship. Since, corrosion of material mainly depends on the interaction between the material and water.[21] Superhydrophobic surfaces shows very less wettability so, they possess very high corrosion resistance.[22] The electrochemical studies are carried out using Gamry reference 600 potentiostat/galvanostat, adopting three electrode system in which platinum foil, saturated calomel electrode are used as counter and reference electrodes. Bare aluminium/superhydrophobic aluminium of $1 \mathrm{~cm}^{2}$ area acted as working electrode. All samples are immersed in $3.5 \% \mathrm{NaCl}$ for 24 hours before the electrochemical experiments to ensure the attainment of stable open circuit potential.

\subsection{Point of zero charge (pzc) determination}

To understand the relationship between $\mathrm{pH}$ and water contact angle, the aluminium substrate was immersed in anhydrous octane (non-polar solvent) which has a almost similar dispersion contribution to the surface free energy $\left(21.6 \mathrm{mJm}^{-2}\right.$ for water and $21.3 \mathrm{mJm}^{-2}$ for octane).[23-25] solutions ranging from $\mathrm{pH} 1$ to $\mathrm{pH} 14$ were prepared. (shown in Fig. 3) by adding hydrocholoric acid and sodium hydroxide.

$\mathrm{pH}$ of the solutions were measured using Hanna Instruments Inc., USA waterproof $\mathrm{pH}$ meter with an accuracy of $\pm 0.01 \mathrm{pH}$. Average five measurements of water contact angle at different places of the substrate in octane medium is measured and average is considered. Ten contact angle measurements were taken per $\mathrm{pH}$ value with the solution volume droplet of $2 \mu \mathrm{L}$ using Kyowa DMs 200, Japan instrument. 


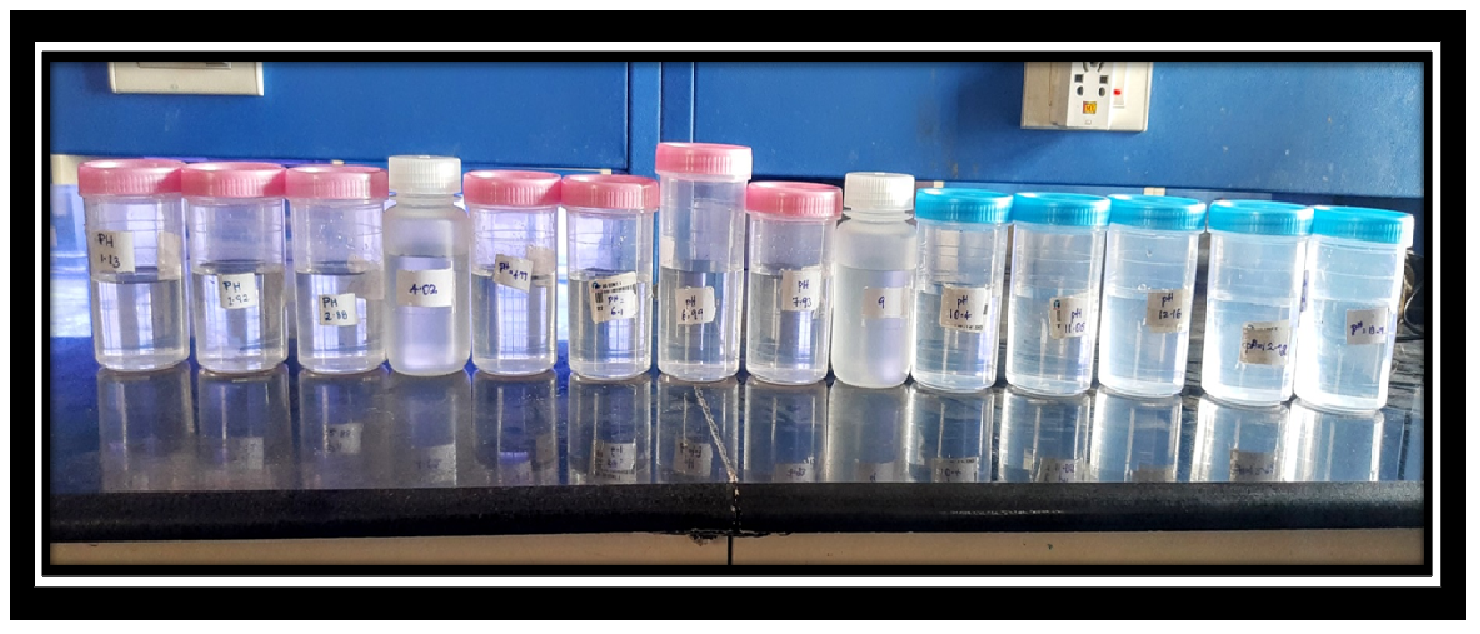

Fig. 3solution of different $\mathrm{pH}$ starting from $\mathrm{pH} 1$ to 14 .

The reference substrate was attempted for contanct angle study using n-octane solution as an immersive medium. A glass tub to hold the substrate and liquid medium was constructed as shown in Fig. 4.
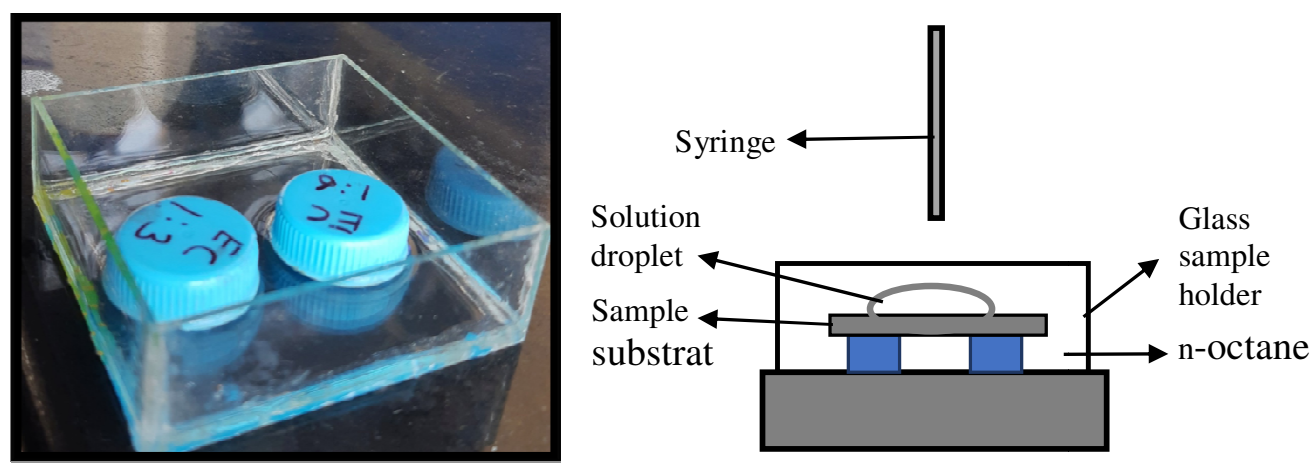

Fig. 4 a) optical image of sample holder b) schematic of contact angle measurement in noctane medium.

\section{Results and Discussion}

\subsection{Surface morphology}

Fig. 3 displays the SEM images of a) and c) displays the SEM image and water contact angle of anodized and sealed aluminium (2.0 $\mathrm{A} \mathrm{dm}^{-2}$ for 30 minutes) substrate. b) and d) displays the SEM image and water contact angle of $\mathrm{SH}$ aluminium substrate. The superhydrophobic aluminium sample showed the WCA greater than $150^{\circ}$. The anodized sealed aluminium $\left(2.0 \mathrm{~A} \mathrm{dm}^{-2}\right.$ for 30 minutes) substrate shows the hydrophilic contact angle $(<$ 
$\left.90^{\circ}\right)$. It is mainly due to the presence of aluminium oxyhydroxide $(\mathrm{AlO}(\mathrm{OH}))$ present on the substrate after anodization and sealing. The superhydrophobic surface shows number of pinshaped projections, out of which few are projecting upwards and others projecting horizontally. From the SEM image the micro-nano arrangement of the lauric acid molecules are clearly seen. The pin-shaped projections point upwards are expected to most effectively reduce the water contact area between the surface and water droplet. As expected the static water contact angle of superhydrophobic surface is increased in comparison with the anodized and sealed aluminium (2.0 $\mathrm{A} \mathrm{dm}^{-2}$ for 30 minutes) substrate.
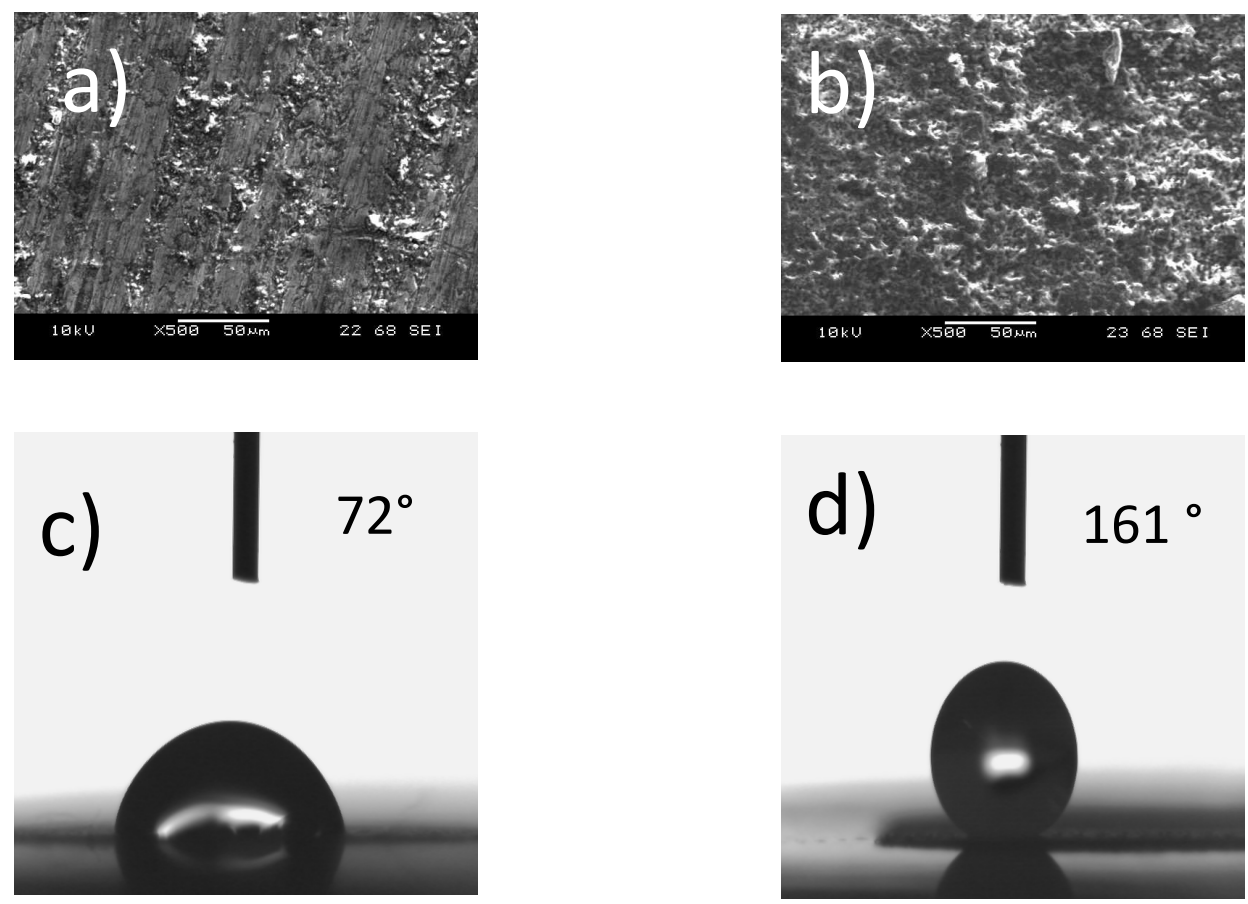

Fig.3 SEM image of (a) anodized and sealed aluminium (2.0 $\mathrm{A} \mathrm{dm}^{-2}$ for 30 minutes) substrate b) superhydrophobic aluminium substrate c) Static water contact angle of bare aluminium substrate (volume of water droplet $\approx 5 \mu \mathrm{L}$ ) d) Static water contact angle of superhydrophobic aluminium substrate. (volume of water droplet $\approx 5 \mu \mathrm{L}$ )

\subsection{Electrochemical impedance spectroscopy (EIS)}

The EIS measurements were done between $10^{6}$ and $10^{-2} \mathrm{~Hz}$ against the open circuit potentials with AC perturbation of $10 \mathrm{mV}$. Using the value of charge transfer resistance $\left(\mathrm{R}_{\mathrm{ct}}\right)$ corrosion inhibition efficiency can be calculated as follows:

$$
I E \%=\frac{R_{c t}^{\prime}-R_{c t}}{R_{c t}^{\prime}} X 100-----e q(1)
$$


In this $R_{c t}^{\prime}$ and $R_{c t}$ are the charge transfer resistance values in the superhydrophobic and bare aluminium substrate in $3.5 \% \mathrm{NaCl}$ medium respectively. The depressed semicircle loop at higher frequencies because of heterogeneity of surface roughness on the electrode surface due to the absorption of inhibitor molecules.[26, 27] The charge transfer resistance gets increased and all the three molecules possess inhibition effects on aluminium corrosion.[28] The experimental results fit well with the equivalent circuit shown in Fig. 4. Constant phase element (CPE) is widely to represent the non-ideal capacitive behavior, the analysis of impedance behavior of all the inhibitors were studied using the following equation:[29]

$$
Z_{c p e}=\frac{1}{Y_{0}(j \omega)^{n}}-----e q(2)
$$

The ideal capacitance value $\left(\mathrm{C}_{\mathrm{dl}}\right)$ was measured from the CPE values using the following expression.[28]

$$
C_{i d}=\frac{Y_{0} \omega^{n-1}}{\sin \left(\frac{n}{2}\right)}-------e q(3)
$$

The maximum value of the angular frequency due to imaginary part $-\mathrm{Z}$ is expressed as ' $\square$ ', direct relationship between the angular frequency and double layer capacitance was given in the following equation:[28]

$$
C_{d l}=Y_{0}(\omega)^{n-1}-----e q(4)
$$

(a)

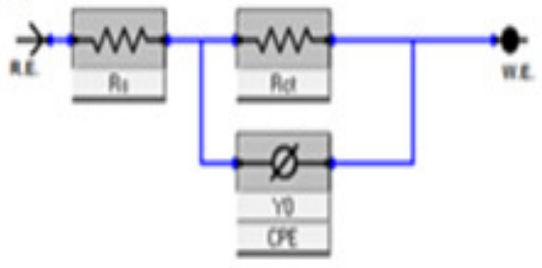

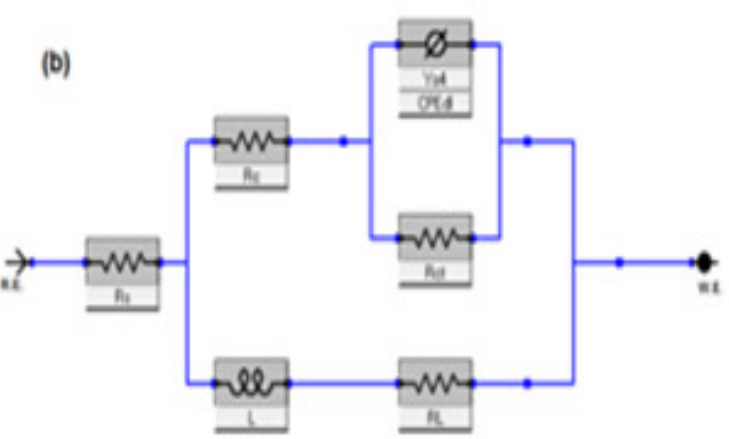

Fig 4: Electrochemical equivalent circuit obtained from EIS studies of (a) bare aluminium and (b) superhydrophobic aluminium 


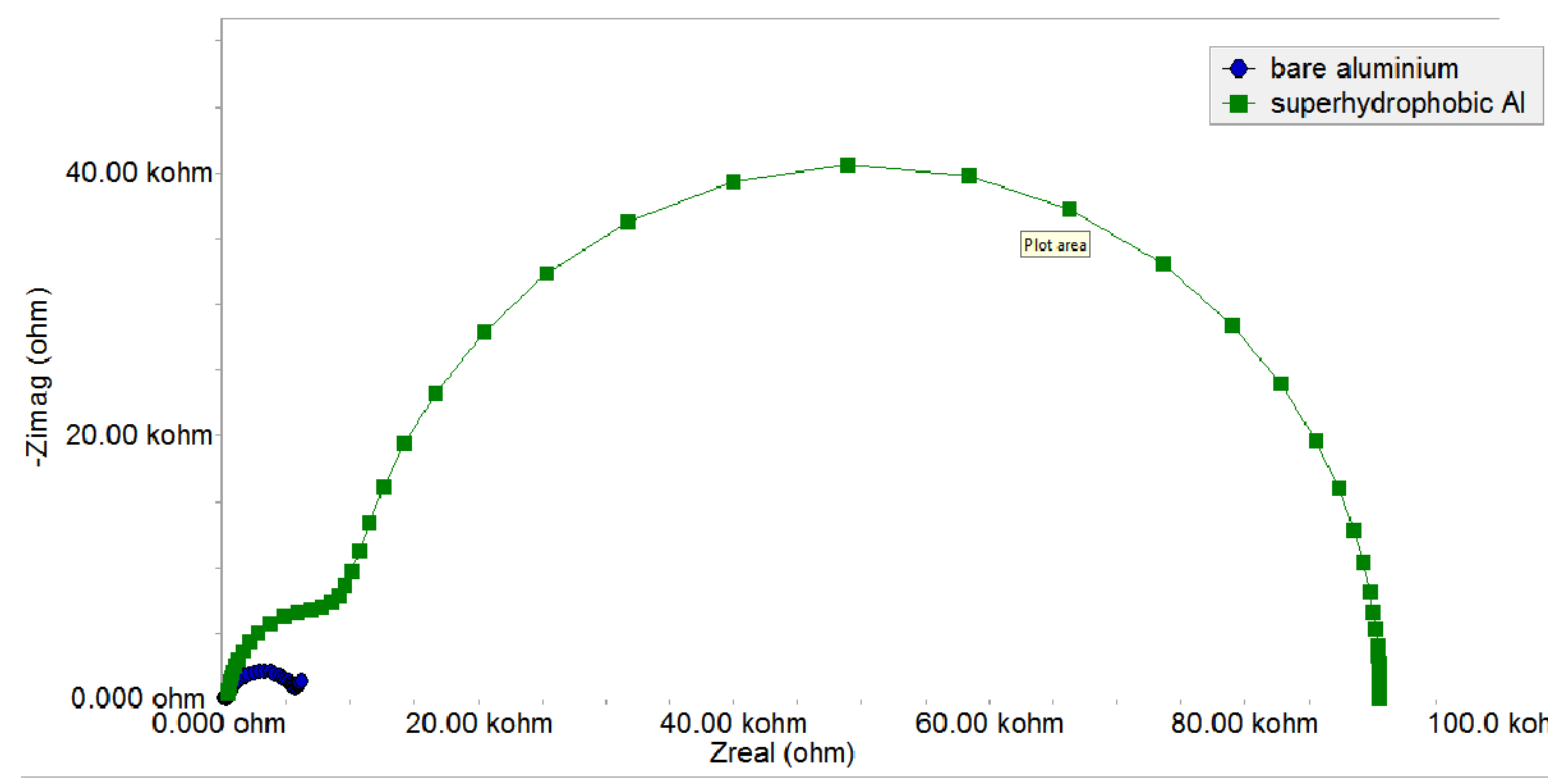

Fig. 5 Electrochemical impedance spectrum of bare aluminium and superhydrophobic aluminium

Table 1: Electrochemical parameters of electrochemical impedance spectroscopy fitted to equivalent circuits.

\begin{tabular}{|c|c|c|c|c|c|c|}
\hline \multirow[t]{2}{*}{ Sample } & \multirow{2}{*}{$\begin{array}{c}R_{\mathrm{ct}}(\mathbf{k} \wedge \\
\left.\mathrm{cm}^{2}\right)\end{array}$} & \multicolumn{2}{|c|}{$\mathrm{CPE}_{\mathrm{dl}}$} & \multirow{2}{*}{$\begin{array}{c}\mathbf{C}_{\mathbf{c}} \\
(\mu \mathrm{F} / \mathrm{c} \\
\left.\mathbf{m}^{2}\right)\end{array}$} & \multirow{2}{*}{$\begin{array}{l}R_{s}(\wedge \\
\left.\mathbf{c m}^{2}\right)\end{array}$} & \multirow{2}{*}{$\begin{array}{r}\mathbf{R}_{\mathrm{c}}(\mathbf{k} \wedge \\
\left.\mathbf{c m}^{2}\right)\end{array}$} \\
\hline & & $\begin{array}{c}Y_{0}\left(\mu \mathrm{F} /\left(\mathrm{cm}^{2}\right.\right. \\
\left.\left.\mathrm{Hz}^{1-\mathrm{n}}\right)\right)\end{array}$ & $\mathbf{n}$ & & & \\
\hline Bare aluminium & 6.4 & 64.24 & 0.90 & - & 180.6 & - \\
\hline Superhydrophobi & & & & & & \\
\hline aluminium & 1470.4 & 0.54 & 0.81 & 3.87 & 210.7 & 0.009 \\
\hline
\end{tabular}

From Fig 5 and Table 1, $\mathrm{R}_{\mathrm{ct}}$ for the substrates are measured in the $3.5 \mathrm{wt} \%$ aqueous solution of $\mathrm{NaCl}$ increases with $\mathrm{SH}$ formation. When immersion duration of aluminium 
substrate in sodium laurate solution, the $\mathrm{i}_{\text {corr }}$ decreases, whereas the $\mathrm{R}_{\mathrm{ct}}$ increases. The $\mathrm{C}_{\mathrm{dl}}$ decreases with formation of superhydrophobicity. So, the (IE\%) can expressed as follows:[29]

$$
I E=\frac{R_{t}-R_{c t}}{R_{t} X 100}
$$

Where $R_{t} \& R_{c t}$ are charge transfer resistance of fabricated superhydrophobic surface on aluminium and bare aluminium respectively. IE of surface after treatment with sodium laurate were estimated to be $99.5 \%$. There will be an increase in $\mathrm{pH}$ near the site of corrosion which is due to the formation of hydroxide ions. This increase in $\mathrm{pH}$ (around 13) to the highly alkaline medium dissolves the superhydrophobic film, [30, 31] the introduction of laurate ion takes place in the corroding environment.

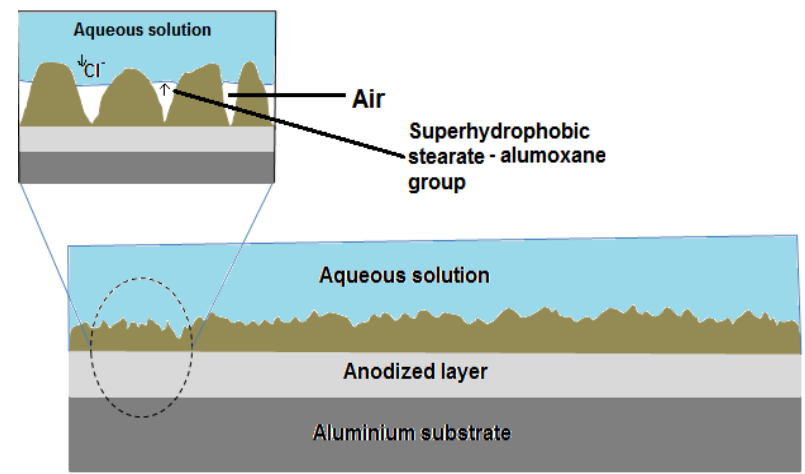

Fig. 6 Schematic representation of solid-liquid interaction of superhydrophobic surface

Fig. 6 the schematic representation of superhydrophobic surface and the corrosive aqueous solution is expressed in Fig. 6. The aluminium substrate after anodization forms a continuous film of aluminium oxide above its surface. The aluminium oxide formed is porous in nature. In order to decrease its porosity and to introduce hydroxyl group $\left(\mathrm{OH}^{-}\right)$on the surface, it is sealed in boiling water for about 30 minutes. After sealing the surface morphology of the aluminium substrate changes. $\mathrm{AlO}(\mathrm{OH})$ is formed after sealing increases the concentration of hydroxyl group on the surface.[32] The point of zero charge of this surface will be based on the amount of hydroxyl group which are projecting out. [33]These hydroxyl group interact with the aqueous solution. During the conversion of superhydrophobic process, the number of hydroxyl group decreases due to the interaction between lauric acid and $\mathrm{AlO}(\mathrm{OH})$. The $\mathrm{H}^{+}$ions present on the lauric acid reacts with the $\mathrm{OH}^{-}$present on $\mathrm{AlO}(\mathrm{OH})$ so that the aluminium laurate chemically grafted on the surface of aluminium. The hydrophobic tail of the laurate group projecting out is responsible for the superhydrophobic nature. The non-polar hydrocarbon chain creates the surface imperfections which is essential for the superhydrophobic nature.[34, 35] The air gets trapped on the valleys which repels the water molecules so, the chance of penetration of chloride ion decreases which results in the increase in corrosion resistance.

\subsection{Potentiodynamic polarization method}


Fig. 7 displays the graphical output of the potentiodynamic polarization studies of bare aluminium and superhydrophobic aluminium in aqueous solution of $3.5 \mathrm{w} / \mathrm{v} \% \mathrm{NaCl}$. A decrease of about three order of magnitude of corrosion current (5.63 $\mu \mathrm{A}$ to $40.0 \mathrm{nA})$ for a superhydrophobic surface when compared with bare aluminium confirms the SHs shows very better resistance against corrosion in aqueous solution of $3.5 \mathrm{w} / \mathrm{v} \% \mathrm{NaCl}$. Corrosion potential $\left(\mathrm{E}_{\text {corr }}\right)$ of the fabricated superhydrophobic surface is shifted to more positive value $(-445.8$ $\mathrm{mV}$ ) when compared with the bare aluminium substrate $(-742.7 \mathrm{mV})$. The corrosion mechanism of any base material were affected by the surface nature and wettability. Greater the wettability, more the corrosion rate and vice versa. So, any superhydrophobic surface with poor wettability is expected to have very good corrosion resistance. The surface of superhydrophobic material will have 'air valleys' which prevents the interaction of water molecule with the coating surface. A convex surface was formed by the water molecule at the junction liquid and air. Our superhydrophobic film contains a large number of $-\mathrm{CH}_{2}$ groups, which results in the shift of $\mathrm{E}_{\mathrm{corr}}$ towards more positive potential by increasing the cathodic current. In addition to this, the anodic and cathodic current density of the superhydrophobic surface decreases considerably due to the decrease in the rate of anodic and cathodic reactions.

Table 2: Electrochemical parameter obtained from potentiodynamic polarization

\begin{tabular}{|c|c|c|c|c|c|c|}
\hline $\begin{array}{c}\text { Corrosion } \\
\text { medium }\end{array}$ & $\begin{array}{l}\text { Concentration of } \\
\text { inhibitor }(\mathbf{m M})\end{array}$ & $\begin{array}{l}\mathbf{E}_{\text {corr }} \\
(\mathbf{m V})\end{array}$ & $\begin{array}{l}\mathbf{I}_{\text {corr }} \\
(\boldsymbol{\mu} \mathbf{A})\end{array}$ & $\begin{array}{c}\beta_{c}(m V \\
\left.\operatorname{dec}^{-1}\right)\end{array}$ & $\begin{array}{c}\beta_{\mathrm{a}}(\mathrm{mV} \\
\left.\operatorname{dec}^{-1}\right)\end{array}$ & $\begin{array}{l}\text { Percentage } \\
\text { efficiency } \\
(\text { PE \%) }\end{array}$ \\
\hline $3.5 \%$ & Bare $\mathrm{Al}$ & -742.7 & 5.63 & 748.4 & 127.4 & - \\
\hline $\mathrm{NaCl}$ & $\begin{array}{c}\text { Superhydrophobic } \\
\text { aluminium }\end{array}$ & -445.8 & 0.04 & 87.40 & 89.4 & 98.9 \\
\hline
\end{tabular}

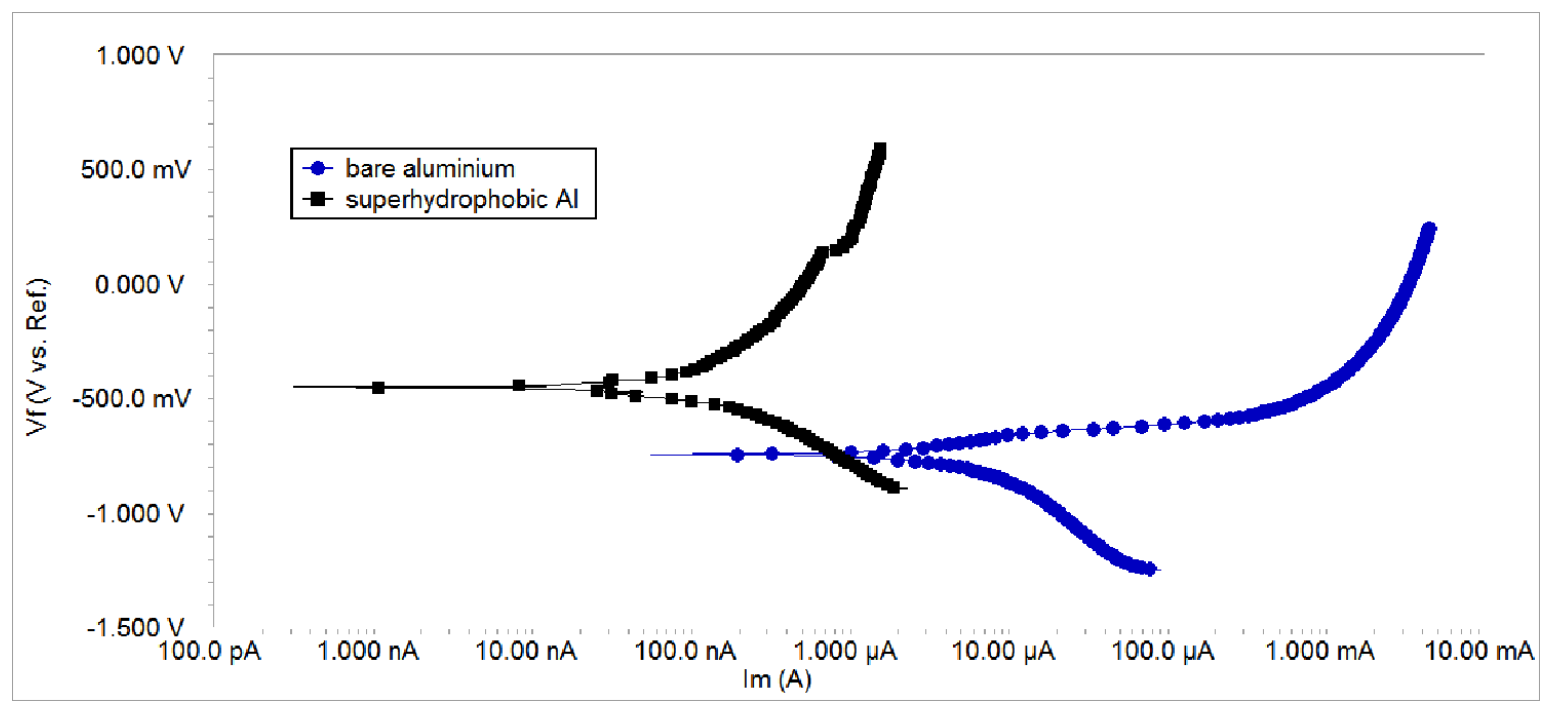


Fig. 7: Potentiodynamic polarization Tafel plots obtained for bare aluminium and superhydrophobic substrate in $3.5 \% \mathrm{NaCl}$

\subsection{Point of zero charge determination}

The anodized and sealed aluminium (2.0 $\mathrm{A} \mathrm{dm}^{-2}$ for 30 minutes) substrate and superhydrophobic aluminium substrate static water contact angle was measured in octane medium using different $\mathrm{pH}$ solution and the values are plotted as graph shown in Fig. 8 .
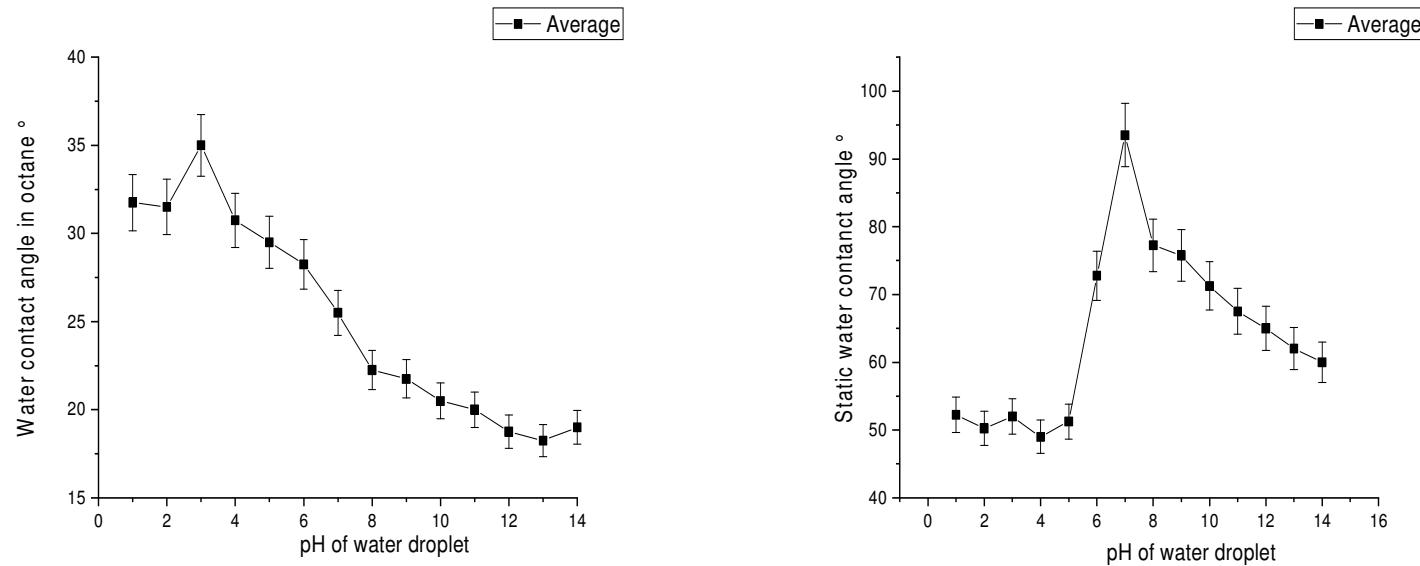

Fig. 8: Graphical representation of WCA in n-octane a) anodized and sealed aluminium b) superhydrophobic aluminium

The maximum static water contact angle which corresponds to the pzc value of the surface and density of hydroxyl group are displayed in Table 1.

\begin{tabular}{lll}
\hline Surface name & WCA $^{\circ}$ & pzc \\
\hline Anodized and sealed aluminium & 35.0 & 3 \\
\hline Superhydrophobic aluminium & 93.5 & 7
\end{tabular}

From the table it is clear that the highest WCA in n-octane is shown by the substrate at $\mathrm{pH}$ of $\approx 3$ on anodized and sealed aluminium substrate and at a $\mathrm{pH}$ of $\approx 7$ on superhydrophobic aluminium substrate. This reveals the pzc of anodized and sealed aluminium and superhydrophobic aluminium are 3 and 7 respectively.

\section{Conclusions}

- Superhydrophobic aluminium substrate was fabricated by adopting simple and economically viable process

- The fabricated surface shows very better resistance against corrosion in aqueous solution of $3.5 \mathrm{w} / \mathrm{v} \% \mathrm{NaCl}$. Three order decrease in corrosion current is visible in SHs. 
- The decrease in corrosion resistance with increase in water immersion time. This is attributed due to the localized corrosion attack on the SH surface. Formation of hydroxide ions takes and the $\mathrm{pH}$ increases at the vicinity of corrosion site.

- A simple procedure using static water contact angle is adopted to determine the pzc. By following this procedure the pzc of anodized and sealed aluminium and superhydrophobic aluminium substrate were determined.

\section{Acknowledge}

The authors thank the management of PSG institutions and Principal of PSG College of technology for their financial support and moral support. Subramanian Sathy Srikandan thanks the financial support provided by UGC - New Delhi. (MRP-5691/15(SERO/UGC).

\section{References}

[1] J. Demaison, M. Herman, J. Liévin, The equilibrium OH bond length, International Reviews in Physical Chemistry, 26 (2007) 391-420.

[2] S. Yamamoto, K. Andersson, H. Bluhm, G. Ketteler, D.E. Starr, T. Schiros, H. Ogasawara, L.G. Pettersson, M. Salmeron, A. Nilsson, Hydroxyl-induced wetting of metals by water at near-ambient conditions, The Journal of Physical Chemistry C, 111 (2007) 7848-7850.

[3] J.S. Noh, J.A. Schwarz, Estimation of the point of zero charge of simple oxides by mass titration, Journal of Colloid and Interface Science, 130 (1989) 157-164.

[4] E. Cristiano, Y.-J. Hu, M. Siegfried, D. Kaplan, H. Nitsche, A comparison of point of zero charge measurement methodology, Clays and Clay Minerals, 59 (2011) 107-115.

[5] J.J. Gulicovski, L.S. Čerović, S.K. Milonjić, Point of zero charge and isoelectric point of alumina, Materials and manufacturing processes, 23 (2008) 615-619.

[6] E. Rossi, P.S. Phani, R. Guillemet, J. Cholet, D. Jussey, W. Oliver, M. Sebastiani, A novel nanoindentation protocol to characterize surface free energy of superhydrophobic nanopatterned materials, Journal of Materials Research, (2021) 1-14.

[7] K. Terpilowski, Surface free energy of superhydrophobic materials obtained by deposition of polymeric particles on glass, Advances in contact angle, wettability and adhesion, 2 (2015) 381-395.

[8] T. Rezayi, M.H. Entezari, F. Moosavi, The variation of surface free energy of Al during superhydrophobicity processing, Chemical Engineering Journal, 322 (2017) 181-187.

[9] J. Bachmann, G. McHale, Superhydrophobic surfaces: a model approach to predict contact angle and surface energy of soil particles, European journal of soil science, 60 (2009) 420-430.

[10] F. Lapierre, Y. Coffinier, R. Boukherroub, V. Thomy, Electro-(de) wetting on superhydrophobic surfaces, Langmuir, 29 (2013) 13346-13351.

[11] R. Mohammadi, J. Wassink, A. Amirfazli, Effect of surfactants on wetting of super-hydrophobic surfaces, Langmuir, 20 (2004) 9657-9662.

[12] E. Virga, E. Spruijt, W.M. de Vos, P.M. Biesheuvel, Wettability of amphoteric surfaces: the effect of $\mathrm{pH}$ and ionic strength on surface ionization and wetting, Langmuir, 34 (2018) 15174-15180.

[13] F.S. Kamelian, T. Mohammadi, F. Naeimpoor, M. Sillanpaa, One-Step and Low-Cost Designing of Two-Layered Active-Layer Superhydrophobic Silicalite-1/PDMS Membrane for Simultaneously 
Achieving Superior Bioethanol Pervaporation and Fouling/Biofouling Resistance, ACS Applied Materials \& Interfaces, 12 (2020) 56587-56603.

[14] M. Ramezani, M.R. Vaezi, A. Kazemzadeh, Preparation of silane-functionalized silica films via two-step dip coating sol-gel and evaluation of their superhydrophobic properties, Applied Surface Science, 317 (2014) 147-153.

[15] M. Nagayama, K. Tamura, Dissolution of the anodic oxide film on aluminium in a sulphuric acid solution, Electrochimica Acta, 12 (1967) 1097-1107.

[16] F. Debuyck, M. Moors, A. Van Peteghem, The influence of the anodization temperature and voltage on the porosity of the anodization layer on aluminium, Materials chemistry and physics, 36 (1993) 146149.

[17] W. Lee, R. Ji, U. Gösele, K. Nielsch, Fast fabrication of long-range ordered porous alumina membranes by hard anodization, Nature materials, 5 (2006) 741-747.

[18] P. Sheasby, R. Guminski, T. Castle, A Quantitative Method of Testing the Quality of Sealing of Anodized Aluminium, Transactions of the IMF, 44 (1966) 50-56.

[19] G. McHale, N. Shirtcliffe, M. Newton, Contact-angle hysteresis on super-hydrophobic surfaces, Langmuir, 20 (2004) 10146-10149.

[20] Y. Xiu, L. Zhu, D.W. Hess, C. Wong, Relationship between work of adhesion and contact angle hysteresis on superhydrophobic surfaces, The Journal of Physical Chemistry C, 112 (2008) 1140311407.

[21] T. Xiang, S. Ding, C. Li, S. Zheng, W. Hu, J. Wang, P. Liu, Effect of current density on wettability and corrosion resistance of superhydrophobic nickel coating deposited on low carbon steel, Materials \& Design, 114 (2017) 65-72.

[22] T. Xiang, D. Chen, Z. Lv, Z. Yang, L. Yang, C. Li, Robust superhydrophobic coating with superior corrosion resistance, Journal of Alloys and Compounds, 798 (2019) 320-325.

[23] E. Chibowski, P. Staszczuk, Determination of surface free energy of kaolinite, Clays and Clay Minerals, 36 (1988) 455-461.

[24] E. Chibowski, L. Hołysz, G.A. Kip, A. van Silfhout, H. Busscher, Surface free energy components of glass from ellipsometry and zeta potential measurements, Journal of colloid and interface science, 132 (1989) 54-61.

[25] D.K. Owens, R. Wendt, Estimation of the surface free energy of polymers, Journal of applied polymer science, 13 (1969) 1741-1747.

[26] F. Mansfeld, Electrochemical impedance spectroscopy (EIS) as a new tool for investigating methods of corrosion protection, Electrochimica Acta, 35 (1990) 1533-1544.

[27] K. Jüttner, Electrochemical impedance spectroscopy (EIS) of corrosion processes on inhomogeneous surfaces, Electrochimica Acta, 35 (1990) 1501-1508.

[28] S. Mirabedini, G. Thompson, S. Moradian, J. Scantlebury, Corrosion performance of powder coated aluminium using EIS, Progress in organic coatings, 46 (2003) 112-120.

[29] A. Simões, D. Battocchi, D. Tallman, G. Bierwagen, Assessment of the corrosion protection of aluminium substrates by a Mg-rich primer: EIS, SVET and SECM study, Progress in Organic Coatings, 63 (2008) 260-266.

[30] H.O. Curkovic, E. Stupnisek-Lisac, H. Takenouti, The influence of $\mathrm{pH}$ value on the efficiency of imidazole based corrosion inhibitors of copper, Corrosion Science, 52 (2010) 398-405. 
[31] S. Xu, Q. Wang, N. Wang, X. Zheng, Fabrication of superhydrophobic green surfaces with good self-cleaning, chemical stability and anti-corrosion properties, Journal of Materials Science, 54 (2019) 13006-13016.

[32] T. Hoar, G. Wood, The sealing of porous anodic oxide films on aluminium, Electrochimica Acta, 7 (1962) 333-353.

[33] D.S. Vijayan, A. Mohan, J. Revathy, D. Parthiban , R. Varatharajan, "Evaluation of the impact of thermal performance on various building bricks and blocks: A review", Environmental Technology \& Innovation 23 (2021) 101577, https://doi.org/10.1016/j.eti.2021.101577.

[34] Z. Lin, Y. Liu, C.-p. Wong, Facile fabrication of superhydrophobic octadecylamine-functionalized graphite oxide film, Langmuir, 26 (2010) 16110-16114.

[35] M. Wolfs, T. Darmanin, F. Guittard, Effect of hydrocarbon chain branching in the elaboration of superhydrophobic materials by electrodeposition of conducting polymers, Surface and Coatings Technology, 259 (2014) 594-598. 